\title{
Performance Comparison of Serial Concatenated Convolutional Codes and Parallel Concatenated Convolutional Codes on Faded Channels
}

\author{
Sajjad Ahmed Ghauri, M Sajid Javaid, Hanan Adeel \\ National University of Modern Languages \\ Islamabad, Pakistan
}

\author{
I.M. Qureshi \\ Air University, \\ Islamabad, Pakistan
}

\begin{abstract}
The performance comparison of Serially Concatenated Convolutional Codes (SCCC) and Parallel Concatenated Convolutional Codes (PCCC) is evaluated on Additive White Gaussian Noise model channel, which is used as a basis, and actions of SCCC and PCCC on Raleigh faded channels are evaluated in this research work. In DCS the SCCC and PCCC are utilized to reduce the bit error rates. SCCC and PCCC encoder consists of RSCC. The major thrust is iterative decoding. This research has utilized BCJR and TDA decoding algorithms. This research analyzed SCCC and PCCC with interleaved scheme. The BPSK modulation was considered. Performance metric for SCCC and PCCC over Memory less AWGN channel, and memory based channels i.e. Rayleigh slow and fast Faded Channel is BER verses SNR.
\end{abstract}

\section{Introduction}

In DCS design, channel encoding enhances the bits in error rate by added redundancy. The data sequences and parity bits are transformed into coded words. (Proakis, 1995). Improved performances are shown with increased encoder's memory in CC. Similarly, for block codes increased codeword length, by the raise in encoder memory size the decoder complexity based on maximum likelihood (ML) would increase. The recent task for coding and Message bit Scientists is, How to design a powerful and practical code with performance close to the Shannon's capacity limit?

This devised scheme is used in construction of Serially Concatenated Convolutional Codes (turbo codes) this approach is applied (Berrou et. al., 1994). "Turbo Codes" (Berrou et. Al., 1993) have emerged as the latest updates in coding theory. Serially Concatenation version of $\mathrm{CC}$ comprises of two RSCC encoders. The information bits passed in to 1st RSCC encoder and, the permuted version of output bit sequence is presented to second RSCC encoder. Using decoding process which is iterative in nature, SCCC achieves low BER which is approximately close to Shannon limit theorem.

In Turbo Decoding Algorithm two decoders are employed, each turn out approximation of symbol. Communication of information between decoders while operating in (SISO) mode. This research is designed and analyzed the SCCC encoding and decoder using BCJR and Turbo Decoding Algorithm.

\section{Methodology}

\subsection{SCCC ENCODER}

The input bit sequence is encoded utilizing two constituent component codes, which are recursive in

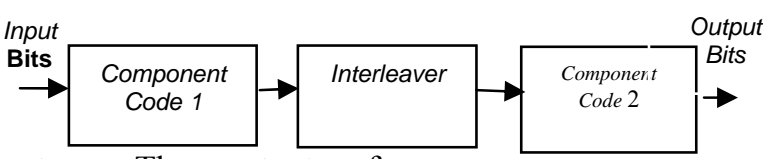

nature. The output of

external encoder is feed to the Inner encoder.

Figure 1. SCCC Encoder

The input to the inner encoder is the interleaved edition of the output of outer encoder.

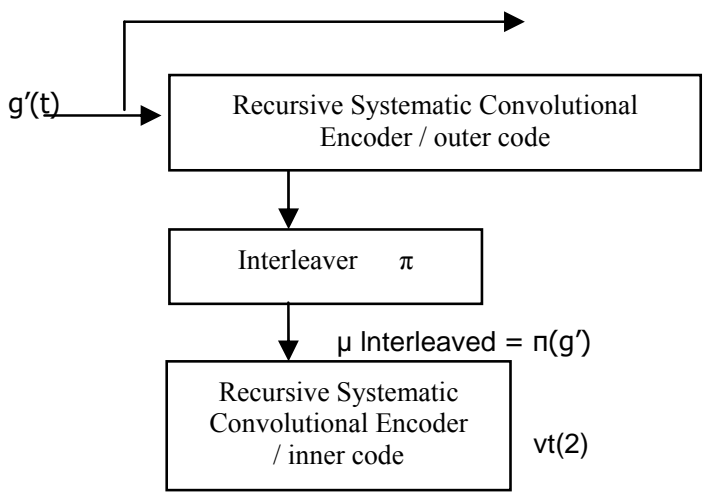

Figure 2. Input/Out

The input symbols at the input of encoder is $\mathrm{g}^{\prime}=\left\{\mathrm{g}^{\prime} 0, \mathrm{~g}^{\prime} 1, \ldots, \mathrm{g}^{\prime} \mathrm{n}-1\right\}$, where each g'i belongs to $\{0,1\}$. After adding zeros the data sequence becomes, $\mathrm{g}=\{\mathrm{go}, \mathrm{g} 1, \ldots, \mathrm{gN}-1\}$. Sequence is equal to the data sequence.

$$
\mathrm{v}_{\mathrm{t}}^{(1)}=\mathrm{g}_{\mathrm{t}}, \mathrm{t}=0,1, \ldots, \mathrm{N}-1 .
$$

Second, the input is fed in RSCC encoder, resulting in a $\{\mathrm{vo}(2), \mathrm{v} 1(2), \ldots, \mathrm{vN}-1(2)\}$. The coded sequence $\{\mathrm{vo}(2), \mathrm{v} 1(2), \ldots, \mathrm{vN}-1(2)\}$ is first fed in to the interleaver which results as ulnter $=\pi\left(v^{\prime}\right)$. 
This interleaved sequence ulnter is fed in to the RSCC encoder, which is, $\operatorname{vt}(3)=\{\operatorname{vo}(3), \mathrm{v} 1(3), \ldots$ $, \mathrm{vN}-1(3)\}$. The outputs of SCCC encoder are code rate $\mathrm{Rv}=1 / 2$.

$\mathrm{V}=\{(\operatorname{vo}(1), \mathrm{v} 0(2)),,(\mathrm{v} 1(1), \mathrm{v} 1(2)),, \ldots \ldots,(\mathrm{vN}-$ $1(1), \mathrm{vN}-1(2))$,$\} .$

The channels may be characterizes with different types of errors. Burst error causes lost to contiguous symbol bits. In account, decoding schemes should be devised. If the conversion and encoding of data occur in such a way that burst error encountered across the channel and seems to be a random error. Interleaving performed at the encoder mitigates the effects of burst error along different fading channels. Fading is a phenomenon which occurs due to time diversity. A number of diversity methods are used to drop down channel impairment effects either by randomizing occurred burst error.

There is minimum correlation among interleaved version of feed bits so burst errors can be converted into random error. Thus burst error may be healed off using existing schemes to cope with random error. Different choices of interleavers include random interleavers, block interleavers along with helical interleaver. In this research, Smart SCCC code utilizes random interleaver. When random interleaver is utilized, it is hard to find the correlation among input bits sequence.

\subsection{PCCC Encoder}

Schematic diagram of PCCC encoder is shown in Figure 3. The input bits are coded using two component codes. Component codes are RSCC.

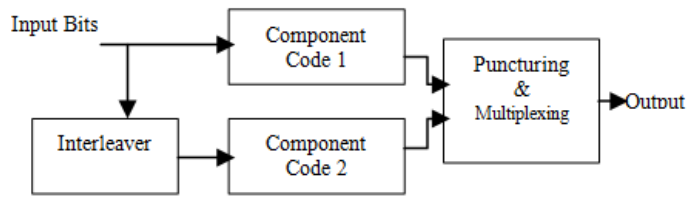

Figure 3. PCCC encoder

RSCC encoders are separated by an interleaver are shown in Figure below. The second RSCC encoder works on a permuted version of the input frame while using Interleaver.

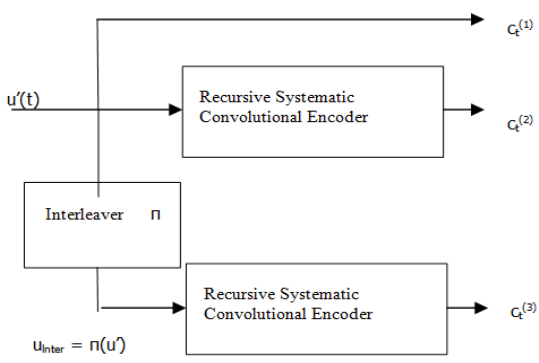

Figure 4. Block Diagram of PCCC encoder
The input symbols block are at the input of encoder is $u^{\prime}=\left\{u^{\prime} o, u^{\prime} 1, \ldots, u^{\prime} n-1\right\}$, where each u'i belongs to alphabet A $\varepsilon\{0,1\}$. After appending zeros the message sequence becomes $\mathrm{u}=\{\mathrm{uo}, \mathrm{u} 1, \ldots$ ., uN-1\}. In the encoder block diagram, the input sequence $u$ ' is used in three ways.

First, coded sequence is exactly equal to the message sequence. $\operatorname{ct}(1)=\mathrm{u}^{\prime} \mathrm{t}, \mathrm{t}=0,1, \ldots, \mathrm{N}-1$. Second, the input sequence fed in to the first RSCC encoder, resulting in a coded plus parity sequence $\{\operatorname{co}(2), \mathrm{c} 1(2), \ldots, \mathrm{cN}-1(2)\}$. Third, the message sequence $u^{\prime}$ is first passed through the interleaver which permutes the message sequence as ulnter $=\pi$ (u'). This interleaved sequence ulnter is passed through RSCC encoder, which produces the output sequences $\operatorname{ct}(3)=\{\operatorname{co}(3), \operatorname{cl}(3), \ldots, \operatorname{cN}-1(3)\}$. The outputs of PCCCencoder are multiplex together, resulting code rate $\mathrm{Rc}=1 / 3$.

$\mathrm{C}=\left\{\left(\mathrm{c}_{\mathrm{o}}{ }^{(1)}, \mathrm{c}_{0}{ }^{(2)}, \mathrm{c}_{\mathrm{o}}{ }^{(3)}\right),\left(\mathrm{c}_{1}{ }^{(1)}, \mathrm{c}_{1}{ }^{(2)}, \mathrm{c}_{1}{ }^{(3)}\right), \ldots \ldots \ldots \ldots\right.$
$\left.\ldots \ldots \ldots,\left(\mathrm{c}_{\mathrm{N}-1}{ }^{(1)}, \mathrm{c}_{\mathrm{N}-1}{ }^{(2)}, \mathrm{c}_{\mathrm{N}-1}{ }^{(3)}\right)\right\}$

Because of interleaving the codes have two sets of parity information $\mathrm{c}(2)$ and $\mathrm{c}(3)$, which are fairly independent. Using puncturing before multiplexing we obtain the higher data rates, as shown in Figure5.

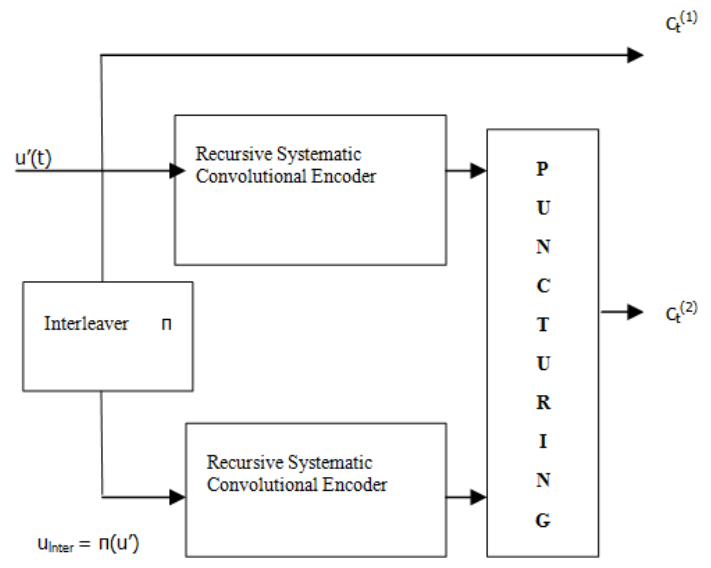

Figure 5. Generalized Block Diagram of PCCC encoder

\subsection{SCCC Decoding}

The decoding for Serially \& Parallel Concatenated Codes is accomplished by two local optimum decoders connected or cascaded to each other (Phillip, 2005). This research utilized both decoders with BCJR algorithm to accomplish the task. The inner and outer decoders are cascaded serially and they exchange the information about the decoded binary digits. Although they are different from PCCC but the BCJR algorithm or forwardbackward algorithm works with serially cascaded codes. This is not heuristic. The secret to application of BCJR to SCCC and PCCC is that there are some systematic changes are made. They are less assumption and more these are systematic changes. The following points are to be kept in mind before 
decoding is done. Firstly, the code rate for inner and outer codes is different. Secondly, posterior probabilities of outer code and inner code are used iteratively to reach a consensus. The SCCC decoding algorithm operation is shown in Figure 6.

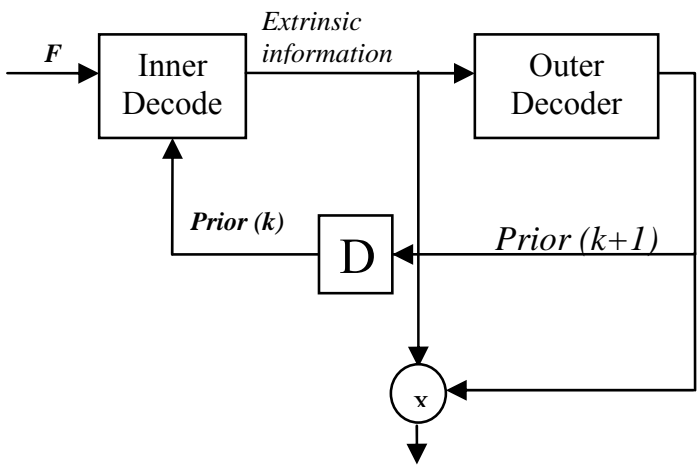

Pseudo posteriors

Figure 6. SCCC decoding algorithm

\subsection{BCJR Algorithm}

BCJR algorithm is also known as forwardbackward algorithm. This decoding algorithm is used in decoding of concatenated or cascade codes. As various versions or factions of cascading are practiced, the BCJR algorithm works for some factions of concatenated codes easily and for some versions with suitable changes or assumptions. The key feature of BCJR or forward-backward algorithm is to reduce decoding complexity and it does a soft decoding. This is no less than Viterbi decoding or Trellis decoding. In BCJR algorithm, intrinsic and extrinsic information is utilized in computing the probability of input binary digits. The detailed example of BCJR algorithm will be presented shortly. The BCJR machinery decodes the received bit sequence.

\section{Convolutional Encoding}

The input data sequence is represented by $\mathrm{m}$.

$m=\{m(1) m(2) \ldots m(J)\}$

Each element of $\mathrm{m}$ consists of $\mathrm{b}$ binary digits or tuples.

$m(j)=m(j, 1) m(j, 2) \ldots m(j, b)$

The size of encoder's memory is denoted by s. The input data sequence should be appended by s 0 's before feeding it to the encoder. The zero appended data sequence is represented by $\mathrm{m}$ '. By appending $\mathrm{s}$ 0 's will leave the encoder in known state. The size of appended data sequence is $\mathrm{J}+\mathrm{s}$.

$m^{\prime}=\{m(1) m(2) \ldots m(J) m(J+1) m(J+2) \ldots m(J+s)\}$
The output of encoder for appended input data sequence is referred as encoded sequence and represented by $\mathrm{w}$. Each element in data sequence $\mathrm{w}$ consists of $\mathrm{B}$ binary digits with $\mathrm{B}>\mathrm{b}$.

$w(j)=w(j, 1) w(j, 2) \ldots w(j, B)$

BPSK modulation is done on the output of the convolutional encoder and is referred as modulated string. Modulated string is represented by $p(t)$. The 0 's in $\mathrm{p}(\mathrm{t})$ are mapped to -1 's and 1's remained unchanged. This mapping may be achieved by this function:

$$
f=\left\{\begin{array}{cl}
1 & p(t, j)=1 \\
-1 & p(t, j)=0
\end{array}\right.
$$

After passing through the channel the sequence is referred as outcome string and is represented by $\mathrm{F}=$ $\{\mathrm{F}(1) \mathrm{F}(2) \ldots \mathrm{F}(\mathrm{J}+\mathrm{s})\}$. The channel noise is represented by $\mathrm{y}(\mathrm{t})$. $\mathrm{y}(\mathrm{t})$ is a Random, $\mathrm{ZM}$ and Gaussian. The time instances are represented by subscripted k. k- represents past time instance and $\mathrm{k}+$ indicates future instance. The encoder may be in one of $\mathrm{P}$ positions. Total possible positions for the encoder are $2 \mathrm{~s}$.

After every time instance, the encoder may be in the same or another position. The sequence of positions is represented by $\mathrm{S}$. S is an array and contents of $\mathrm{S}$ represents the positions followed by time instances. The size of array $\mathrm{S}=\mathrm{M}$, indeed $\mathrm{M}=$ $\mathrm{J}+\mathrm{s}$. We have used $\mathrm{k}$ to represent the subscript of the array. So, $s(k)$ represents the position of encoder at time instance, $\mathrm{k}$. The array may be initialized by $\{0\}$. This leads that the initial position of the encoder is an all cleared position. The change in position of the encoder is referred as position switching. The position switches from position $\mathrm{p} 1$ to $\mathrm{p} 2$. Where $\mathrm{p} 1$ and $\mathrm{p} 2$ belongs to $\mathrm{P}$ and $\mathrm{p} 1 \neq \mathrm{p} 2$ is not necessary. The specific input which results in position switching in response of encoder is denoted as $\mathrm{m}^{\prime}(\mathrm{p} 1, \mathrm{p} 2)$. In result of this position switching, the corresponding mapping symbols are represented as $\mathrm{p}(\mathrm{p} 1, \mathrm{p} 2)$.

$p=\{p(1, \mathrm{p} 1, \mathrm{p} 2) \mathbf{p}(2, \mathrm{p} 1, \mathrm{p} 2) \mathbf{p}(3, \mathrm{p} 1, \mathrm{p} 2) \ldots \mathbf{p}(q, p 1, p 2)\}$

\subsection{Posterior Probability}

At the receiver end two operations are done; detection and decoding. The position-switching are stored in array $\mathrm{S}$ and is utilized in soft decoding procedure. The decoder transverses the array and approximates the sent bits. In order to accomplish this task, BCJR algorithm eminently utilizes the probability calculations of position switching to another position in order to find the analogous probability of sent bits which is now related to the contents of array S.

The position switching is a Markov Process. A Markov Process is associated with random variables, the chance that encoder will be in a particular future 
position, at a specific instance, depends only on the current position and does not depends on any precedent positions. In connection to the statement passed, the exponential outcome sequence is partitioned in three classes, $F=F^{-}+F^{\prime}+\mathbf{F}^{+}$. Where $\mathrm{F}$ - represents signal before time instance $\mathrm{k}$. Similarly $\mathrm{F}$ ' represents signal at time $\mathrm{k}$ and $\mathrm{F}+$ represents signal after time $\mathrm{k}$.

$$
P(S(k), S(k+1) \mid F)=\frac{P(S(k), S(k+1), F)}{P(F)}
$$

Eq. 1

$=\frac{P\left(S(k), S(k+1), F-, F^{\prime}\right) P\left(F+\mid S(k), S(k+1), F-, F^{\prime}\right)}{P(F)}$

Eq. 2

$=\frac{P\left(S(k+1), F^{\prime} \mid S(k), F-\right) P(S(k), F-) P(F+\mid S(k+1))}{P(F)}$

Eq. 3

By markovity Eq. 3 becomes,

$=\frac{P\left(S(k+1), F^{\prime}\right) P\left(S(k+1), F^{\prime} \mid S(k)\right) P(F+\mid S(k+1))}{P(F)}$

Eq. 4

In Eq. 4 the joint probability $\mathrm{P}(\mathrm{S}(\mathrm{k}), \mathrm{F}-)$ is denoted by $\mathrm{A}(\mathrm{k}, \mathrm{p} 1)$.

$$
\mathrm{A}(k, p 1)=P\left(S(k+1), F^{\prime}\right)
$$

Eq. 5

Similarly,

$\zeta(k, p 1, p 2)=P\left(S(k+1), F^{\prime} \mid S(k)\right)$

Eq. 6

$Z(k+1, p 2)=P(F+\mid S(k+1))$

Eq. 7

The posterior probability for position switching

$P(S(k), S(k+1) \mid F)=\frac{A(k, p 1), \zeta(k, p 1, p 2), Z(k+1, p 2))}{P(F)}$

Eq. 8

\subsubsection{Recursive Algorithm to find A's}

If the Posterior probabilities of position switching are known then it leads to compute the posterior probability of the binary digit $\mathrm{p}\left(\mathrm{m}^{\prime}(\mathrm{k})=\mathrm{m}^{\prime} \mid \mathrm{F}\right)$. For every data $m$ ' in set $\alpha$, the possible position switches $\mathrm{p} 1, \mathrm{p} 2$ matching to $\mathrm{m}^{\prime}(\mathrm{k})=\mathrm{m}^{\prime}$ are represented by Tm.

$$
\operatorname{Tm}=\left\{(\mathrm{p} 1, \mathrm{p} 2) \in \mathrm{P} \mid \mathrm{m}^{\prime}(\mathrm{p} 1, \mathrm{p} 2)=\mathrm{m}^{\prime}\right\}
$$

The posterior probability of $\mathrm{m}^{\prime}(\mathrm{k})=\mathrm{m}$ can be determined by adding position switches $\forall$ members of Tm .
$P\left(m^{\prime}(k)=m^{\prime} \mid F\right)=\sum_{(p 1, p 2) \in S m^{\prime}} P(S(k), S(k+1) \mid F)$

Eq. 9

$P\left(m^{\prime}(k)=m^{\prime} \mid F\right)=\sum_{(p 1, p 2) \in S m^{\prime}} \frac{A(k, p 1) \zeta(k, p 1, p 2) Z(k+1, p 2)}{p(F)}$

Eq. 10

Conditional probabilities $A(k+1, p 2)$ are computed utilizing assumption as $A(k, p 1)$ are known to us $\forall$ positions, $\mathrm{P}$.

$$
A(k+1, p 2)=P\left(S(k+1=p 2), F^{-}(k+1)\right)
$$

Eq. 11

$$
A(k+1, p 2)=P\left(S\left(k+1=p 2, F(k), F^{-}(k)\right)\right)
$$

(by Definition)

Let us find the Marginal probabilities

$A(k+1, p 2)=\sum_{p 1} P\left(S(k+1)=p 2, F(k), S(k)=p 1, F^{-}(k)\right)$
$A(k+1, p 2)=\sum_{p 1} P\left(S(k+1)=p 2, F(k) \mid F^{-}(k)\right) P\left(S(k)=p 1, F^{-}(k)\right)$ Eq. 12

Utilizing conditioning factorization

$A(k+1, p 2)=\sum_{p 1} P\left(S(k)=p 1, F^{-}(k)\right) P\left(S(k+1)=p 2, F^{-}(k) \mid S(k)=p 1, F^{-}(k)\right)$

By definition of $\mathrm{A}(\mathrm{k}, \mathrm{p} 1)^{\zeta}(\mathrm{k}, \mathrm{p} 1, \mathrm{p} 2) \&$ Markovity

$A(k+1, p 2)=\sum_{p 1} P\left(S(k)=p 1, F^{-}(k)\right) P\left(S(k+1)=p 2, F(k) \mid F^{-}(k)\right)$

Eq. 13

$A(k+1, p 2)=\sum_{p 1} A(p 1) \zeta(k, p 1, p 2)$

Eq. 14

In Trellis pass A-Probabilities are regarding the forward pass; means they are initiated from start on initial set, $\mathrm{A}(0, \mathrm{p} 1)$ with $\mathrm{p} 1 \in \mathrm{P}$ (total possible positions for the encoder).

\subsubsection{Recursive Algorithm to Find Z's}

A good estimation of $\mathrm{Z}(\mathrm{k}, \mathrm{p} 2)$, probabilities associated with the backward pass, is possible if $\mathrm{Z}$ ( $\mathrm{k}+1, \mathrm{p} 2)$ are priory known $\forall$ positions in which the convolutional encoder can be. This recursive definition of Z's is known as backward pass. The procedure to find recursive relationship for Z's is demonstrated here:

$Z(k, p 1)=p\left(F^{+}(k-1) \mid S(k)=p 1\right)$

Eq. 15

$Z(k, p 1)=p\left(F^{+}(k), F(k) \mid S(k)=p 1\right)$

With definition for F Marginal form of probabilities from Joint form yields, 
$Z(k, p 1)=\sum_{p 2} p\left(F^{+}(k), S(k+1)=p 2 \mid S(k)=p 1\right)$

Eq. 16

$Z(k, p 1)=\sum_{p 2} p(F(k), S(k+1)=p 2 \mid S(k)=p 1) p\left(F^{+}(k) \mid F(k), S(k+1)=p 2\right), S(k)=$

By means of $\mathrm{Z}(\mathrm{k}+1, \mathrm{p} 2) \zeta(\mathrm{k}, \mathrm{p} 1, \mathrm{p} 2) \&$ Markovity definitions, we have

$\left.Z(k, p 1)=\sum_{p 2} p(F(k), S(k+1)=p 2 \mid S(k)=p 1) p\left(F^{+}(k) \mid S(k+1)=p 2\right)\right)$

Eq. 17

$Z(k, p 1)=\sum_{p 2} \zeta(k, p 1, p 2) Z(k+1, p 2)$

Eq. 18

\subsubsection{Finding Probabilities $\zeta(k)$}

Now, after finding the recursive relationships for forward and backward passes, i.e. A's and Z's, need to compute $\zeta$ 's as these are used in both probabilities. $\zeta$ 's denotes the position switching probabilities. Method to find these position switching probabilities is illustrated below by means of mathematical equations.

$\zeta(k, p 1, p 2)=p(S(k+1)=p 2, F(k) \mid S(k)=p 1)$ Eq. 19

$\zeta(k, p 1, p 2)=p(F(k), S(k)=p 1, S(k+1)=p 2) p S(k+1)=p 2 \mid S(k)=p 1)$ 19(b)

If it is known that the encoder is in position $\mathrm{p} 1$ at time instance say $\mathrm{k}$, and encoder keeps position $\mathrm{p} 2$ at time instance $\mathrm{k}+1$, identifies the output $\mathrm{P}(\mathrm{t})$ which was represented by $\mathrm{f}(\mathrm{p} 1, \mathrm{p} 2)$ and matching input $\mathrm{m}^{\prime}(\mathrm{p} 1, \mathrm{p} 2)$.

$p(S(k+1)=p 2 \mid S(k)=p 1)=p\left(m(k)=m^{\prime}(p 1, p 2\right.$ Eq. 20

The probability $p\left(m(k)=m^{\prime}(p 1, p 2)\right.$ is known as apriori. This probability is flat and equals to a half $(1 / 2)$ or 0.5 in binary coded signaling. This assumption helps in simplifying the calculations. In d-dim AWGN communication channel this probability happen to be Gaussian shaped.

$p(F(k) \mid f(p 1, p 2))=\frac{1}{\sqrt[d]{2 \pi} \sigma} \exp \left(\frac{1}{2 \sigma^{2}}\|F(k)-f(p 1, p 2)\|^{2}\right.$

Eq. 21

Plugging values from Eq. 20 and Eq. 21 in Eq. 19 (b), we get for BPSK modulation.

$\zeta(p 1, p 2)=\frac{1}{\sqrt[d]{2 \pi} \sigma} \exp \left(\frac{1}{2 \sigma^{2}}\|F(k)-f(p 1, p 2)\|^{2}\right) p\left(m^{\prime}(k)=m^{\prime}(p 1, p 2)\right)$

Eq. 22

\subsubsection{Initial Assignments for A's and Z's pass}

The initial assignments for A's and Z's are done in this way. As the encoder memory elements are all cleared at start so $\mathrm{p} 1=0.0$ indicates that all memory $p$ Eontents are cleared. So probability factor in position 0 for $\mathrm{A}(\mathrm{k}=0)$ are initialized as

$\left(\mathrm{A}(0,1) \mathrm{A}(0,2) \ldots \mathrm{A}(0, \mathrm{~T})=\left(\begin{array}{llll}1 & 0 & \ldots & 0\end{array}\right)\right.$

Eq. 23

Where $A(a, b)$ indicates time instance which represented by $\mathrm{k}$ and $\mathrm{b}$ indicates the position of the encoder so $\mathrm{b}$ runs from 1 to $\mathrm{T}, \mathrm{T}$ indicates total positions in the switching string.

In the same fashion, the initial assignments for Z's are done in this way. As the encoder memory elements finishes in a known position i.e. all cleared position so initialize

$\left(\mathrm{Z}(\mathrm{M}, 1) \mathrm{Z}(\mathrm{M}, 2) \ldots \mathrm{Z}(\mathrm{M}, \mathrm{T})=\left(\begin{array}{llll}1 & 0 & \ldots & 0\end{array}\right)\right.$

Eq. 24

Where $\mathrm{M}$ is the size of $\mathrm{S}$,

$P\left(m^{\prime}(k)=m^{\prime} \mid F\right)=\sum_{p 1, p 2 \in S_{m} \cdot} \frac{A(k, p 1), \zeta(k, p 1, p 2), Z(k+1, p 2))}{P(F)}$

Eq. 25

\subsubsection{PCCC Decoding Algorithm}

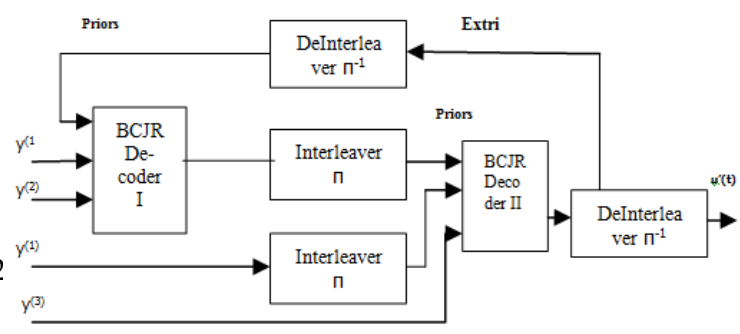

Figure 7. PCCC Decoding Algorithm

The coded sequence which is punctured is transmitted over a noisy channel (AWGN \& Rayleigh Fading). The received sequence $\mathrm{y}$ is demultiplexed into sequences $\mathrm{y}(1)$ corresponding to $\mathrm{c}(1), y(2)$ corresponding to $\mathrm{c}(2)$ and $\mathrm{y}(3)$ corresponding to $\mathrm{c}(3)$.

The PCCC decoding algorithm operation is shown in Figure. The received sequence $\mathrm{y}(1), \mathrm{y}(2)$ (consociated with the first encoder) are fed into decoder-I. The decoder-I produces probabilities of bits which are conditioned on the observed data, probabilities of bits are referred as extrinsic probabilities. The interleaved version of output of decoder-I are fed in to the decoder-II, where output of decoder-I are used as "prior" probabilities, along which is $\mathrm{y}(1)$ (interleaved) and $\mathrm{y}(2)$ are as an input to decoder-II. The extrinsic probabilities of decoder-II 
are de-interleaved and passed back to become prior probabilities to decoder-I. The process of communicating information probability back and forth continues until maximum number of iterations is accomplished or till the decoder specifies that decoding process is converged.

\section{Analysis}

The Figure below shows the performance comparison of PCCC and SCCC on faded channel and SCCC performs better in terms of BER as compared to PCCC, the BER of SCCC at $\mathrm{SNR}=10 \mathrm{~dB}$ is $10-6$ and $\mathrm{PCCC}$ is $10-4$.

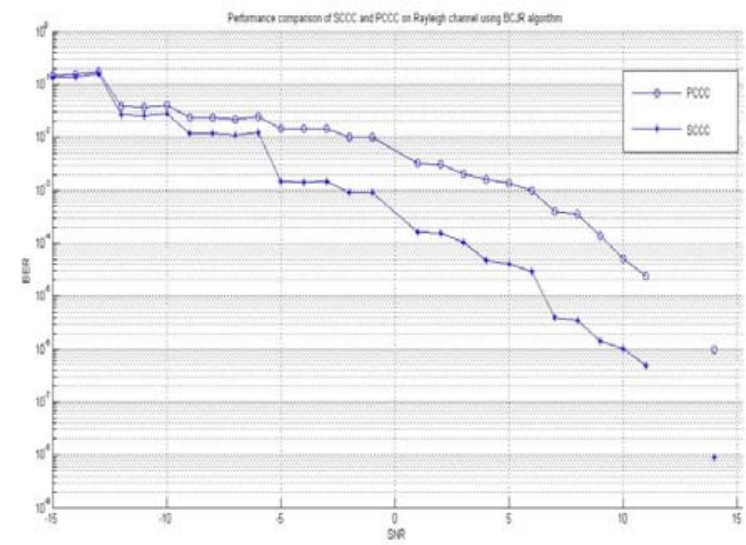

Figure 8. Performance Comparison of SCCC and PCCC on Rayleigh Faded Channel using BCJR Algorithm

The Figure 9 shows the performance of PCCC and SCCC on Rayleigh Faded channel using TDA and simulation results shows SCCC performs better as compared to PCCC.

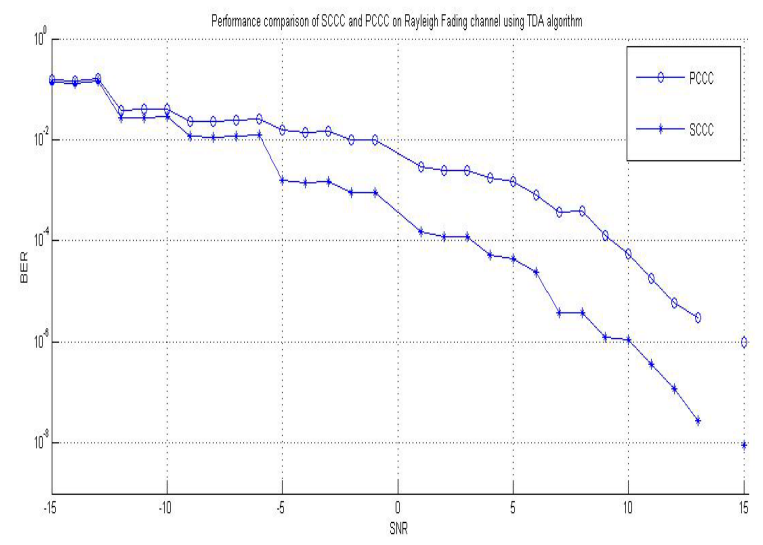

Figure 9. Performance Comparison of SCCC and PCCC on Rayleigh Faded Channel using TDA

The main title (on the first page) should begin 1$3 / 8$ inches $(3.49 \mathrm{~cm})$ from the top edge of the page, centered, and in Times 14-point, boldface type. Capitalize the first letter of nouns, pronouns, verbs, adjectives, and adverbs; do not capitalize articles, coordinate conjunctions, or prepositions (unless the title begins with such a word). Leave two 12-point blank lines after the title.

\section{Conclusion}

"Performance comparison of SCCC \& PCCC on faded Channels", conferred iterative decoder with SCCC. Smart SCCC \& PCCC coding and decoding algorithm has executed close to Shannon capacity. Thus, the bits in error rate of these SCCC codes is much improved than their PCCC counter codes. A variable forward error control system can be implemented with puncturing and rate-compatibility.

The computer based simulation results show that SCCC is an influential forward error correction coding technique that works with channels having certain SNR circumstances. There are featuring factors needed to be careful in Smart SCCC coding $\&$ decoding. As the decoding process is iterative, so yielding lower BER is conditioned to number of iterations. In order to drop the BER, if increases the iterations, it will affect the decoding latency. Further, the bit in error or BER surrenders provided the frame size larger. The proceeding in this manner also effects the decoding time. Moreover, another key factor to be considered in decoding procedure is code rate. The bandwidth needs increases for higher coding rate. The chief hitch of SCCC is their complex design, and decoding time. The simulation results also show that SCCC performs better under Fading channels as compared to PCCC.

\section{References}

[1] Bahl, "Optimal Decoding of Linear Codes for Minimizing Symbol Error Rate," IEEE Trans. Inf. Theory, vol. IT-20, pp. 284-287, Mar. 1974.

[2] Berrou 1994, "Turbo-codes and high spectral efficiency modulation" in Proc. ZCC '94, New Orleans, LA, May 1994

[3] C. Berrou , 1993, "Near Shannon limit error-correcting coding and decoding: Turbo-codes," in Proc. ZCC'Y3, Geneva, Switzerland, May 1993, pp. 1064-1070

[4] C. Berrou, 1993, "Turbo codes: General principles and Applications" in Proc. 6th Tirrenia Int. Workshop Digital Communication, Tirrenia, Italy, Sept. 1993

[5] Forney G. D. Concatenated Codes. Cambridge, MA: MIT Press, 1966

[6] Kousa M. A. 2002, "Puncturing effects on Turbo Codes," IEEE Proceedings Communications, Vol.149, 2002, pp.132138

[7] M. Awad, K. T. Wong \& Z. Li, An Integrative Overview of the Open Literature's Empirical Data on the Indoor Radiowave Channel's Temporal Properties, IEEE Transactions on Antennas \& Propagation, vol. 56, no. 5, pp. 1451-1468, May 2008.

[8] S. Le Goff, "Turbo-codes and high spectral efficiency modulation," in Proc. ZCC '94, New Orleans, LA, May 1994

[9] Sklar B., "Rayleigh Fading Channels In Mobile Digital Communication Systems", IEEE Communication Magazine. July 1997

[10] Viterbi A. J. 1967 , "Error Bounds for Convolutional Codes and an Asymptotically Optimum Decoding Algorithm," IEEE Trans. Inf. Theory, IT-13, April 1967, pp. 260-269. 